\title{
Kinetics of Presynaptic Filament Assembly in the Presence of SSB and Mediator Proteins ${ }^{\dagger}$
}

\author{
Jie Liu $\ddagger$, I, Christopher L. Berger§, and Scott W. Morrical ${ }^{\ddagger},{ }^{*}$ \\ ₹Department of Biochemistry, University of Vermont College of Medicine, Burlington, VT 05405 \\ $\S$ Department of Molecular Physiology and Biophysics, University of Vermont College of Medicine, \\ Burlington, VT 05405
}

\section{Abstract}

Enzymes of the RecA/Rad51 family catalyze DNA strand exchange reactions that are important for homologous recombination and for the accurate repair of DNA double-strand breaks. RecA/ Rad51 recombinases are activated by their assembly into presynaptic filaments on single-stranded DNA (ssDNA), a process that is regulated by ssDNA-binding (SSB) and mediator proteins. Mediator proteins stimulate strand exchange by accelerating the rate-limiting displacement of SSB from ssDNA by the incoming recombinase. The use of mediators is a highly conserved strategy in recombination, but the precise mechanism of mediator activity is unknown. In this study, the welldefined bacteriophage T4 recombination system (UvsX recombinase, Gp32 SSB, and UvsY mediator) is used to examine the kinetics of presynaptic filament assembly on native ssDNA in vitro. Results indicate that the ATP-dependent assembly of UvsX presynaptic filaments on Gp32covered ssDNA is limited by a salt-sensitive nucleation step in the absence of mediator. Filament nucleation is selectively enhanced and rendered salt-resistant by mediator protein UvsY, which appears to stabilize a pre-nucleation complex. This mechanism potentially explains how UvsY promotes presynaptic filament assembly under physiologically relevant conditions of ionic strength and Gp32 concentration. Other data suggest that presynaptic filament assembly involves multiple nucleation events, resulting in many short UvsX-ssDNA filaments or clusters, which may be the relevant form for recombination in vivo. Together, these findings provide the first detailed kinetic model for presynaptic filament assembly involving all three major protein components recombinase, mediator, and SSB, on native ssDNA.

\section{Keywords}

Recombination; presynaptic filament; kinetics; fluorescence; mediator; ssDNA-binding protein

The RecA/Rad51 family of proteins is highly conserved, and its members play central roles in homologous recombination-dependent DNA repair, in support of stalled/collapsed replication forks. RecA/Rad51 enzymes catalyze DNA strand exchange, which is the pairing and physical transfer of strands between homologous DNA molecules. Strand exchange requires the formation of a presynaptic filament consisting of recombinase cooperatively bound to single-stranded DNA (ssDNA $\left.{ }^{\ddagger}\right) .{ }^{1}$ Errors in presynaptic filament assembly cause

\footnotetext{
${ }^{\dagger}$ Supported by NIH Grant nos. GM48847 to S.W.M. and HL63798 to C.L.B., respectively. J.L. was supported by a DOE-EPSCoR predoctoral fellowship in structural biology.

"To whom correspondence should be addressed at: Department of Biochemistry, University of Vermont College of Medicine, 89 Beaumont Avenue, Burlington, VT 05405. smorrica@uvm.edu; Phone number: +1 802-656-8260.

TCurrent address: Department of Microbiology and Molecular Genetics, University of California-Davis, Davis, CA 95616
} 
genome instability and sensitivity to DNA damaging agents. ${ }^{2}$ Humans with defective presynapsis are predisposed to cancer. ${ }^{3}$

The formation of recombinase-ssDNA filaments is regulated by other proteins including ssDNA-binding proteins (SSBs) and recombination mediator proteins (RMPs) ${ }^{4}$ Both protein classes are highly conserved at the functional level. Biochemical studies of different recombination systems have revealed a common mechanism of presynapsis in which (1) an SSB binds to and removes secondary structure from ssDNA and, (2) an RMP then mediates loading of recombinase onto the SSB-ssDNA complex with concomitant release of SSB. ${ }^{4-6}$ The RMP stimulates DNA strand exchange by accelerating the rate-limiting displacement of SSB from ssDNA by the incoming recombinase.

Studies of the bacteriophage T4 recombination system have provided important insights on the biochemical mechanism of presynaptic filament assembly and the nature of RMP function. ${ }^{4-14}$ UvsX protein, the RecA/Rad51 ortholog of T4 phage, exhibits both ssDNAdependent ATPase and DNA strand exchange activities. Gp32, the phage SSB, binds tightly and cooperatively to ssDNA. UvsY, the phage RMP, stimulates UvsX activities by overcoming Gp32 inhibition and promoting its assembly onto ssDNA. Protein requirements for DNA strand exchange activity vary as a function of ionic conditions. ${ }^{7,} 14,15$ Under lowsalt conditions, UvsX and Gp32 proteins are sufficient for in vitro strand exchange, while UvsY is dispensable. However, under high-salt conditions that approximate in vivo ionic strength, UvsY is absolutely required for strand exchange along with UvsX and Gp32. In vivo, $u v s Y$ and $u v s X$ mutants are equally deficient in recombination and repair functions, indicating that the mediator activity of UvsY is essential for UvsX biological function. ${ }^{16,17}$ Previous studies revealed that UvsY successively destabilizes Gp32-ssDNA and stabilizes UvsX-ssDNA interactions. ${ }^{8}{ }^{9}$ Results suggest that UvsY-induced changes in ssDNA structure play a major role in modulating the ssDNA-binding activities of UvsX and Gp32. ${ }^{7-9}$

RMP-dependent assembly of recombinase filaments on SSB-covered ssDNA is a common feature of all recombination systems, but the mechanism by which filaments nucleate and propagate on SSB-ssDNA is poorly understood. To address this problem we developed a real-time assay for the kinetics of $\mathrm{T} 4$ presynaptic filament assembly on Gp32-covered ssDNA, based on changes in the fluorescence of a fluorescein-Gp32 conjugate. ${ }^{6}$ In the current study, we use this assay to determine kinetic parameters for the nucleation and growth of UvsX filaments on Gp32-ssDNA, as functions of salt, UvsY, and nucleotide ligands. The data show that UvsY selectively enhances filament nucleation by stabilizing a salt-sensitive pre-nucleation complex, allowing UvsX assembly and Gp32 displacement to occur at higher salt concentrations. The displacement of Gp32 from ssDNA by incoming UvsX appears to be an active process requiring the ATP-bound form of the recombinase. Other results suggest that short UvsX-ssDNA filaments resulting from multiple nucleation events are sufficient for recombination. Collectively, the data shed new light on the mechanism of presynaptic filament assembly during homologous recombination, and the critical role of recombination mediator proteins in this conserved pathway.

\section{MATERIALS AND METHODS}

\section{Reagents}

T4 proteins and M13mp18 ssDNA were prepared as described. ${ }^{6}$ All ssDNA concentrations are given in nucleotide residues. 6-iodoacetamidofluorescein-labeled Gp32 protein (Gp32F

\footnotetext{
$¥$ Abbreviations: ssDNA, single-stranded DNA; SSB, single-stranded DNA binding protein; RMP, recombination mediator protein; ATP $\gamma$ S, adenosine-5'-(O)-(3-thio)triphosphate.
} 
$€$ ) was prepared as described. ${ }^{6}$ Buffer A contained $20 \mathrm{mM}$ Tris- $\mathrm{HCl}$, $\mathrm{pH} 7.4,3 \mathrm{mM} \mathrm{MgCl}$, and $\mathrm{NaCl}$ as indicated.

\section{Stopped-flow fluorescence assays}

Rapid kinetics assays were performed in a $\mu$ SFM-20 stopped-flow fluorometer (BioLogic Science Instruments, Claix, France) having a $1.2 \mathrm{~ms}$ dead time. This instrument was used for all measurements of Gp32F-ssDNA association kinetics, and for initial studies of Gp32F displacement from ssDNA by UvsX \pm UvsY. All protein and ssDNA concentrations reported are final concentrations after mixing. Reactions were carried out at $25^{\circ} \mathrm{C}$ in Buffer A plus $\mathrm{NaCl}$ as indicated. Excitation wavelength was $460 \mathrm{~nm}$ with a $5 \mathrm{~nm}$ slit. Emission was monitored at wavelengths $>495 \mathrm{~nm}$ using a long-pass-cutoff filter and a $20 \mathrm{~nm}$ slit. Each data trace is the average of 10 independent reactions.

Gp32F-ssDNA association kinetics were modeled according to Scheme 1, which parallels the scheme derived previously for native Gp32. ${ }^{18}$ The fluorescence traces were fitted to double exponentials according to Eq. 1.

$$
F(t)=F_{\infty}-A_{1} \exp \left(-k_{o b s 1} t\right)-A_{2} \exp \left(-k_{o b s 2} t\right) \quad(\text { Eq. 1) }
$$

where $F(t)$ is the fluorescence intensity at time $t, F_{\infty}$ is the fluorescence intensity at infinite time, $A_{1}$ and $A_{2}$ are the amplitudes of the first and second relaxation processes, respectively, and $k_{o b s 1}$ and $k_{o b s 2}$ are the observed rate constants of the first and second relaxation process, respectively. The standard deviations of $k_{o b s 1}$ and $k_{o b s 2}$ reflect the associated uncertainties or fluctuations.

The observed rate constant of the first (fast) relaxation process from the double exponential (Eq. 1) was fitted into Eqs. 2 and 3 to obtain the apparent forward/reverse rate constants and to calculate the equilibrium association constant for $\mathrm{Gp} 32 \mathrm{~F}$ cluster formation on the ssDNA lattice.

$$
\begin{gathered}
k_{\mathrm{obs} 1}=k_{2}(\mathrm{app}) \times[\mathrm{ssDNA}]+k_{-2}(\mathrm{app}) \quad \text { (Eq. 2) } \\
K_{2}=k_{2}(\mathrm{app}) / k_{-2}(\mathrm{app}) \quad \text { (Eq. 3) }
\end{gathered}
$$

\section{Gp32F displacement assays for presynaptic filament assembly}

The kinetics of Gp32F displacement from ssDNA by UvsX \pm UvsY were studied in a Quantamaster QM-6 steady-state fluorometer (PTI, NJ). The QM-6 yielded higher sensitivity and little information loss compared to identical experiments in a BioLogic $\mu$ SFM-20 stopped-flow fluorometer, due to the slow reaction rates involved. Excitation wavelength was $460 \mathrm{~nm}$ with $1 \mathrm{~nm}$ slit, and emission wavelength was $519 \mathrm{~nm}$ with a $5 \mathrm{~nm}$ slit. A long-pass-cutoff filter at $495 \mathrm{~nm}$ was used for emission data collection. The effects of dilution, solution change, photobleaching, inner filter effects, and intrinsic protein fluorescence on experimental data were evaluated as described, ${ }^{6}$ and were found to be negligible under the experimental conditions employed. Starting solutions containing 0.5 $\mu \mathrm{M} \mathrm{Gp} 32 \mathrm{~F}$ and $3.5 \mu \mathrm{M}$ ssDNA were pre-incubated at $25^{\circ} \mathrm{C}$ in Buffer A plus $\mathrm{NaCl}$ and

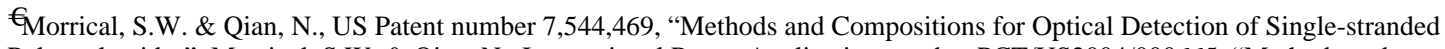
Polynucleotides". Morrical, S.W. \& Qian, N., International Patent Application number PCT/US2004/000665, "Methods and Compositions for Optical Detection of Single-stranded Polynucleotides".
} 
nucleotides as indicated. ATP-containing reactions also contained an ATP-regenerating system ( $5 \mathrm{mM}$ phosphoenolpyruvate, 2.6 units $/ \mathrm{mL}$ pyruvate kinase). UvsX \pm UvsY proteins were separately preincubated in the same buffer/nucleotide solution then manually mixed into the starting solution to initiate the reaction and data collection (dead-time $\approx 3 \mathrm{~s}$ ). The final concentrations of UvsX and UvsY in each experiment are indicated. Emission traces were collected at 3 points/s. Reported observed rate constants are averages obtained from 2 3 separate reactions.

\section{Data analysis}

All fluorescence traces were fitted to double exponentials using Datafit (Oakdale Engineering, PA) according to Eq. 4

$$
F(t)=F_{\infty}-A_{1} \exp \left(-k_{o b s 1} t\right)+A_{2} \exp \left(-k_{o b s 2} t\right) \quad \text { (Eq. 4) }
$$

where $F(t)$ is the fluorescence intensity at time $t, F_{\infty}$ is the fluorescence intensity at infinite time, $A_{1}$ and $A_{2}$ are the amplitudes of the first and second relaxation processes, respectively, and $k_{o b s 1}$ and $k_{o b s 2}$ are the observed rate constants of the first and second relaxation process, respectively. The standard deviations of $k_{o b s 1}$ and $k_{o b s 2}$ reflect the associated uncertainties or fluctuations.

Kinetic traces of Gp32F displacement by UvsX under various conditions were fitted to Eq. 4 to obtain observed rate constants for a fast fluorescence-increasing phase $\left(k_{\mathrm{obs} 1}\right)$ and a slow fluorescence-decreasing phase $\left(k_{\mathrm{obs} 2}\right)$. Both observed rate constants exhibit a hyperbolic dependence on UvsX concentration. A simplified mechanistic model for Gp32F displacement from ssDNA by UvsX is shown in Scheme 2, with two separated phases, filament nucleation $\left(K_{1}, k_{2}\right)$ and filament growth $\left(K_{3}, k_{4}\right)$. This simplified model is consistent with the two-step model (nucleation and growth) obtained from a recent single molecule study of RecA filament formation on SSB-covered ssDNA. ${ }^{19}$ This model makes the simplifying assumptions that under the experimental conditions used: (1) All UvsX is saturated with ATP (or ATP $\gamma \mathrm{S}$ ); (2) isomerization steps $k_{2}$ and $k_{4}$ are slow and essentially irreversible; and (3) all UvsX is bound to UvsY (when present) and corresponding kinetic parameters are those of the UvsX-UvsY complex (stoichiometry undefined). Kinetic parameters in Scheme 2 were estimated by fitting $k_{\mathrm{obs} 1}$ and $k_{\mathrm{obs} 2}$ values obtained at different UvsX concentrations to Eqs. 5 and 6, respectively:

$$
\begin{aligned}
& k_{o b s 1}=k_{2} \times \frac{K_{1}[\mathrm{UvsX}]}{1+K_{1}[\mathrm{UvsX}]}+k_{-2} \quad \text { (Eq. 5) } \\
& k_{o b s 2}=k_{4} \times \frac{K_{3}[\mathrm{UvsX}]}{1+K_{3}[\mathrm{UvsX}]}+k_{-4} \quad \text { (Eq. 6) }
\end{aligned}
$$

\section{Global fitting method in kinetic data analysis}

As an independent method for estimating kinetic parameters, kinetic traces obtained at different UvsX concentrations were globally fit to differential equations derived from the model in Scheme 3, using DynaFit (BioKin Ltd., WA). ${ }^{20}$ Scheme 3 is identical to Scheme 2 but contains term substitutions to facilitate mathematical modeling. The differential equations derived from Scheme 3 are shown in Equations 7.1 - 7.6:

$$
\mathrm{d}[\mathrm{S} 1] / \mathrm{d} t=-k_{1}[\mathrm{~S} 1][\mathrm{S} 2]+k_{-1}[\mathrm{~N} 1]-k_{3}[\mathrm{~S} 1][\mathrm{N} 2]+k_{-3}[\mathrm{~F} 1] \quad(\mathrm{Eq} .7 .1)
$$




$$
\begin{gathered}
\mathrm{d}[\mathrm{S} 2] / \mathrm{d} t-k_{1}[\mathrm{~S} 1][\mathrm{S} 2]+k_{-1}[\mathrm{~N} 1] \quad \text { (Eq. 7.2) } \\
\mathrm{d}[\mathrm{N} 1] / \mathrm{d} t=+k_{1}[\mathrm{~S} 1][\mathrm{S} 2]-k_{-1}[\mathrm{~N} 1]-k_{2}[\mathrm{~N} 1] \quad \text { (Eq. 7.3) } \\
\mathrm{d}[\mathrm{N} 2] / \mathrm{d} t=+k_{2}[\mathrm{~N} 1]-k_{3}[\mathrm{~S} 1][\mathrm{N} 2]+k_{-3}[\mathrm{~F} 1] \quad \text { (Eq. 7.4) } \\
\mathrm{d}[\mathrm{F} 1] / \mathrm{d} t=+k_{3}[\mathrm{~S} 1][\mathrm{N} 2]-k_{-3}[\mathrm{~F} 1]-k_{4}[\mathrm{~F} 1] \quad \text { (Eq. 7.5) } \\
\mathrm{d}[\mathrm{F} 2] / \mathrm{d} t=+k_{4}[\mathrm{~F} 1]
\end{gathered}
$$

In reactions containing UvsY, all UvsX is assumed to be complexed with UvsY, since the two proteins are pre-incubated together and are known to interact strongly under the range of buffer conditions used in these experiments. Therefore [S1] becomes the concentration of free UvsX-UvsY complex, and all other UvsX-containing terms likewise include UvsY. Scheme 3 (Scheme 2) further assumes that due to the experimental conditions: (1) UvsX is saturated with ATP (or ATP $\gamma \mathrm{S}$ ) since nucleotide concentration is higher than $K_{\mathrm{m}}$ or $K_{\mathrm{d}}{ }^{22,23}$, and since experiments with ATP employ a regenerating system to maintain a high ATP/ADP ratio. (2) Free ssDNA in the system is negligible (all of the ssDNA is covered either with Gp32F or with UvsX/UvsY). This assumption is reasonable since Gp32F binds stoichiometrically to mixed sequence ssDNA under the range of buffer conditions used in these experiments ${ }^{6}$, and the concentration of $\mathrm{Gp} 32 \mathrm{~F}$ is saturating with respect to total ssDNA concentration. (3) Steps $k_{2}$ and $k_{4}$ are essentially irreversible $\left(k_{-2}=k_{-4}=0\right)$ so that once Gp32F is displaced from ssDNA it does not compete with UvsX for ssDNA binding sites. This assumption derives from the pre-steady-state conditions including the fact that UvsX \pm UvsY is added to a pre-assembled Gp32F-ssDNA complex, such that the concentration of free $\mathrm{Gp} 32 \mathrm{~F}$ is essentially zero at early stages of each reaction and therefore cannot compete. Also it was observed empirically that the re-occupation of ssDNA by Gp32F requires the hydrolytic depletion of ATP by UvsX, which is prevented in these reactions either by using a regenerating system, or by using $\mathrm{ATP} \gamma \mathrm{S}$.

During global fitting using DynaFit, iterative runs for individual isotherms were taken to determine the values and standard errors of rate constants $k_{2}, k_{4}, k_{1}$, and $k_{3}$ sequentially, according to Eq. 7. To facilitate convergence, rate constants $k_{-1}$ and $k_{-3}$ were each assigned a value of $1 \mathrm{~s}^{-1}$ and were held constant in all iterations, therefore only the association constants for the equilibrium steps $K_{1}\left(=k_{1} / k_{-1}\right)$ and $K_{3}\left(=k_{3} / k_{-3}\right)$ are reported in Table 3 . $K_{1}$ and $K_{3}$ values determined at $50 \mathrm{mM} \mathrm{NaCl}$ are similar in magnitude to estimates of UvsXssDNA binding affinity under equilibrium binding conditions [4; H. Xu and S. Morrical, unpublished results]. The fitting quality can be judged by Figure 4 and by the standard errors of the kinetic constants in Table 3. In nearly all cases the hyperbolic and global fitting methods yielded similar estimates of kinetic parameters $K_{1}, k_{2}, K_{3}$, and $k_{4}$.

\section{RESULTS}

\section{Kinetic properties of Gp32F-ssDNA interactions}

Fluorescein-conjugated Gp32 (Gp32F) exhibits a fluorescence increase upon binding to ssDNA, and its equilibrium ssDNA-binding properties are a reasonable approximation of unlabeled Gp32. ${ }^{6}$ These properties make Gp32F a useful real-time probe for T4 presynaptic filament assembly. To establish the kinetic studies of UvsX-ssDNA assembly on Gp32- 
covered ssDNA, we first investigated the basic kinetic properties of Gp32F-ssDNA binding using stopped-flow fluorescence. Gp32F-ssDNA binding follows the same three-step mechanism previously derived for unlabeled Gp32 (Scheme 1, Figure 1A): ${ }^{18} 1$ ) A very fast nucleation step (too fast to detect) with small amplitude where Gp32F nucleates onto the lattice and forms isolated binding sites. 2) A fast growth step with large amplitude where Gp32F propagates onto the lattice and forms contiguous clusters. 3) A slow isomerization step with small amplitude where the contiguous Gp32F protomers redistribute by facilitated diffusion to form stable clusters on the lattice.

Figure 1A shows a typical kinetic trace when Gp32F is rapidly mixed with excess ssDNA. The time-dependent fluorescence change of $\mathrm{Gp} 32 \mathrm{~F}$ fits a double exponential function (Eq. 1) similar to wild-type Gp32. ${ }^{18}$ The rate constant of the fast phase, $k_{\mathrm{obs}}$, was assigned to the growth step (Step 2, Scheme 1) based on its large amplitude. To obtain rate constants we performed a series of rapid binding experiments in different salt conditions, with constant Gp32F and variable ssDNA concentrations. In low-salt, strong binding conditions (50 - 100 $\mathrm{mM} \mathrm{NaCl}$ ), the observed growth rate constant $k_{\text {obs } 1}$ (Step 2 in Scheme 1) increases with increasing salt concentration (Figure 1B). In high-salt, weak binding conditions (100 - 250 $\mathrm{mM} \mathrm{NaCl}$ ), $k_{\mathrm{obs} 1}$ decreases with increasing salt concentration (Figure 1C). The salt effects on Gp32F-ssDNA binding kinetics parallel those of unlabeled Gp32: ${ }^{18}$ Under low salt conditions $k_{\text {obs } 1}$ increases with increasing salt because $\mathrm{Na}^{+}$disrupts electrostatic interactions between the C-terminal and core domains of Gp32 that inhibit DNA binding. ${ }^{27}$ Under high salt conditions $k_{\mathrm{obs} 1}$ decreases with increasing salt since higher salt interrupts protein-DNA electrostatic interactions.

As shown in Figure 1B-C, $k_{\text {obs1 }}$ exhibits linear dependence on [ssDNA] in all salt conditions. Apparent on and off rate constants for the growth step, $k_{2}$ (app) and $k_{-2}$ (app), were obtained from linear fits of these data to Eq. 2. The apparent association rate has a much stronger dependence on $[\mathrm{NaCl}]\left(\mathrm{d} \log k_{2}(\mathrm{app}) / \mathrm{d} \log [\mathrm{NaCl}]=-0.75 \pm 0.01\right)$ than the apparent dissociation rate $\left(\mathrm{d} \log k_{-2}(\mathrm{app}) / \mathrm{d} \log [\mathrm{NaCl}]=-0.19 \pm 0.01\right)$, when increasing salt from 100 to $250 \mathrm{mM} \mathrm{NaCl}$. In the same salt interval, the equilibrium affinity of $\mathrm{Gp} 32 \mathrm{~F}, K_{\mathrm{ss}}$, dropped from $1.6 \times 10^{5}$ to $7.1 \times 10^{4} \mathrm{M}^{-1}$, and the $\operatorname{d} \log K_{\mathrm{ss}} / \mathrm{d} \log [\mathrm{NaCl}]$ value is equal to $-0.83 \pm 0.03$. The substrate for Gp32F filament assembly is natural phage ssDNA under no tension, and our results indicate the salt impact on the electrostatic interactions between Gp32 and ssDNA is through the decrease of association rate, not increase of dissociation rate.

The rate constants and calculated association constants (Eq. 3) are summarized in Table 1 to compare with unlabeled Gp32. Gp32F displays a 50-fold decrease in $k_{2}$ (app) for binding M13mp18 ssDNA, compared to unlabeled Gp32 binding poly(r\&A). ${ }^{18}$ We previously reported that $\mathrm{Gp} 32 \mathrm{~F}$ shows a 12 -fold decrease in intrinsic binding affinity for M13mp18 ssDNA compared to unlabeled Gp32. ${ }^{6}$ Additionally, Gp32 displays a lattice bias favoring homopolymers and etheno-modified bases (J. Liu and S.W. Morrical, unpublished result). Overall, the data indicate that Gp32F-ssDNA binding follows the same kinetic mechanism (Scheme 1) as unlabeled Gp32, and that the lower affinity of Gp32F vs. Gp32 for ssDNA is largely due to its slower growth on-rate. For these reasons, following the UvsX-dependent displacement of Gp32F from pre-assembled Gp32F-ssDNA complexes provides relevant information on the kinetics of presynaptic filament assembly on native Gp32-ssDNA.

\section{Presynaptic filament assembly as a function of salt, nucleotide, and UvsY}

UvsX-ssDNA filament assembly was monitored by the displacement of Gp32F from ssDNA with concomitant fluorescence decrease. ${ }^{6}$ Pre-assembled Gp32F-ssDNA complexes were rapidly mixed with a stoichiometric amount of UvsX in Buffer A plus $50 \mathrm{mM} \mathrm{NaCl}$ and various nucleotides (Figure 2A). Under these conditions filament assembly is UvsY- 
independent, due to differential salt effects on Gp32-ssDNA versus UvsX-ssDNA interactions (see Discussion). This property is shown clearly by the data in Figure 2A, wherein the addition of UvsX to pre-formed Gp32F-ssDNA in the absence of UvsY leads to a time-dependent decrease in Gp32F fluorescence. The displacement of Gp32F requires ATP binding but not ATP hydrolysis by UvsX, since signal change occurs in the presence of either ATP or ATP $\gamma \mathrm{S}$, but not in the presence of ADP, AMP, or no nucleotide (Figure 2A). The substrate analog ATP $\gamma$ S is very slowly hydrolyzed by UvsX, and stabilizes UvsXssDNA interactions. ${ }^{21,22}$ Note that ATP and ATP $\gamma \mathrm{S}$, when present, were at saturating concentrations, ${ }^{22,23}$ and that an ATP regenerating system was included to maintain a high ATP/product ratio throughout the timecourse. The latter measure was necessary to prevent UvsX dissociation from ssDNA caused by the build-up of ATP hydrolytic products, which eventually leads to the re-occupation of ssDNA by Gp32F. ${ }^{6}$

Increasing salt concentration inhibits presynaptic filament assembly in the absence of UvsY (Figure 2B). All of the reactions in Figure 2B were performed in the presence of saturating ATP plus an ATP regenerating system. Increasing the $\mathrm{NaCl}$ concentration from 50 to 100 $\mathrm{mM}$ causes a sharp decrease in the rate of Gp32F displacement, while $200 \mathrm{mM} \mathrm{NaCl}$ blocks the reaction entirely. The addition of UvsY protein restores filament assembly at $200 \mathrm{mM}$ $\mathrm{NaCl}$, however (Figure 2B). These observations are consistent with salt and UvsY effects on the in vitro DNA strand exchange activity of UvsX protein. ${ }^{7,} 14$ The data may also explain why $\mathrm{T} 4$ recombination transactions are co-dependent on UvsX and Uvs Y in vivo, ${ }^{17}$ where ionic strength is relatively high.

\section{Filament nucleation is rapid but salt-sensitive}

Kinetic traces in the presence of nucleoside triphosphate and at low salt concentration (Figure 2) were fitted accurately by a double exponential function with increasing and decreasing phases (Eq. 4) to obtain the observed rate constants for the fast reaction with small positive amplitude (designated as UvsX nucleation, $k_{\mathrm{obs} 1}$ ) and for the slow reaction with large negative amplitude (designated as UvsX growth, $k_{\mathrm{obs} 2}$ ), as listed in Table 2. In 50 $\mathrm{mM} \mathrm{NaCl}, \mathrm{ATP} \gamma \mathrm{S}$ enhances UvsX's observed nucleation rate about 11 - fold, and observed growth rate about 4 - fold, compared to the natural substrate ATP (Figure 2A and Table 2). This is consistent with the notion that ATP $\gamma \mathrm{S}$ stabilizes the high-affinity ssDNA-binding conformation associated with the ATP-bound form of UvsX.21, 22 The data indicate that this form of UvsX is necessary for Gp32 displacement during both nucleation and growth phases of filament assembly. Note that observed nucleation rates are fast compared to growth rates (Table 2), suggesting that presynaptic filaments assemble from many nucleation centers on Gp32-ssDNA (see Discussion).

In the presence of ATP, doubling the $\mathrm{NaCl}$ concentration from 50 to $100 \mathrm{mM}$ causes a 2.3fold decrease in the observed nucleation rate $\left(k_{\mathrm{obs} 1}\right)$ but has little effect ( $\sim 1.1$-fold decrease) on the observed growth rate (Table 2). Therefore salt has a strong effect on nucleation and a weak effect on growth. Thus the inability of UvsX (+ATP) to displace Gp32F from ssDNA in $200 \mathrm{mM} \mathrm{NaCl}$ (Figure 2B) likely reflects a failure of UvsX to nucleate onto Gp32FssDNA. The addition of UvsY is required for Gp32F displacement under these conditions (Figure 2B). The UvsY-dependent trace is also fit accurately by a double exponential with increasing and decreasing phases (Eq. 4). The observed nucleation rate, $k_{\mathrm{obs}} 1$, of the UvsYdependent reaction in $200 \mathrm{mM} \mathrm{NaCl}$ is 2.4 - and 5.6-fold higher, respectively, than those of the UvsY-independent reactions in 50 and $100 \mathrm{mM} \mathrm{NaCl}$ (Table 2). ATP-binding by UvsX is still required for the UvsY-dependent reaction, however, since no reaction is observed with ADP, AMP, or no nucleotide under these conditions (reference ${ }^{6}$ and data not shown). 


\section{Unique fluorescence signature of filament nucleation}

As shown in Figure 2B, the kinetic trace of the reaction containing UvsY shows a significant initial fluorescence increase, which could be fit accurately to Eq. 4. We attribute the initial fluorescence increase to the formation of a pre-nucleation complex with enhanced Gp32F fluorescence (see Discussion). This pre-nucleation complex is formed in the absence of UvsY as well, since these reactions are also fit accurately by Eq. 4 (Figure 2A-B). In buffer containing $200 \mathrm{mM} \mathrm{NaCl}$, where UvsX alone fails to displace Gp32F from ssDNA, the presence of UvsY increases the observed nucleation rate $\left(k_{\mathrm{obs} 1}\right)$ by at least 470 -fold over the background rate (Table 2$)$. The observed growth rate $\left(k_{\mathrm{obs} 2}\right)$ is 40 -times slower than the observed nucleation rate (Table 2), suggesting that UvsY functions primarily to enhance nucleation rather than growth of UvsX filaments on Gp32-covered ssDNA.

\section{Kinetic model for presynaptic filament assembly}

A series of Gp32F displacement assays were conducted at different UvsX concentrations under high- $(200 \mathrm{mM} \mathrm{NaCl})$ or low-salt $(50 \mathrm{mM} \mathrm{NaCl})$ conditions, and in the presence of either ATP $\gamma$ S or ATP. In $200 \mathrm{mM} \mathrm{NaCl}$ a constant amount of UvsY (stoichiometric w.r.t. ssDNA assuming a binding site size $n=4$ nucleotide residues per monomer ${ }^{24}$ was preincubated with variable amounts of UvsX. Under all conditions the observed rate constants of both nucleation and growth steps appear to increase with increasing UvsX concentration in a saturable manner (Figure 3 ). The data can be approximated using a relatively simple four-step model (Scheme 2): 1) A rapid binding step $\left(K_{1}\right)$ where UvsX binds Gp32F-ssDNA and forms a transient pre-nucleation complex (Gp32F retained). 2) A slow and essentially irreversible isomerization step $\left(k_{2}\right)$ where UvsX changes conformation to form a stable nucleation site (Gp32F displaced). 3) Another rapid binding step $\left(K_{3}\right)$ where UvsX forms unstable contiguous clusters (Gp32F retained). 4) Another slow and essentially irreversible isomerization step $\left(k_{4}\right)$ where UvsX clusters redistribute into stable filaments on the lattice (Gp32F displaced). Steps 1 and 2 comprise the nucleation phase defined by $k_{\mathrm{obs} 1}$ and Eq. 5, whereas steps 3 and 4 comprise the growth phase defined by $k_{\mathrm{obs} 2}$ and Eq. 6 . The assumptions underlying this model are stated in Materials and Methods. Our kinetic model through ensemble studies also agrees well with previous nucleation-growth models from single molecule studies of RecA filament formation on dsDNA or SSB-covered ssDNA. ${ }^{19,25}$

Estimates of $K_{1}, k_{2}, K_{3}$, and $k_{4}$ were obtained by fitting hyperbolic Eqs. 2 and 3, respectively, to plots of $k_{\mathrm{obs} 1}$ or $k_{\mathrm{obs} 2}$ vs. UvsX concentration (Figure 3). The kinetic mechanism in Scheme 2 was tested independently via global fits of model-derived differential equations (Eq. 7) to raw fluorescence trace data (Figure 4). Kinetic parameters determined from both hyperbolic and global fitting methods are listed in Table 3. In nearly all cases the two fitting methods gave very similar results ( $\leq \sim 2$-fold difference in parameter values; Table 3), indicating that Scheme 2 is a reasonable model for this system. The only exception was a 4.6-fold difference in $K_{1}$ obtained from global vs. hyperbolic fitting methods for reactions in $200 \mathrm{mM} \mathrm{NaCl}$ plus UvsY and ATP $\gamma \mathrm{S}$ (Table 3). Under these conditions the global fitting method failed to converge at intermediate UvsX concentrations $(0.1-0.5 \mu \mathrm{M})$, indicating additional complexity in the kinetic mechanism (see Discussion).

Three major findings emerge from the data in Table 3: First, in reactions at $200 \mathrm{mM} \mathrm{NaCl}$, the presence of UvsY strongly and specifically increases $K_{1}$ of the nucleation phase compared to UvsY-independent reactions at $50 \mathrm{mM} \mathrm{NaCl}$. The average increase is 27 -fold with ATP, and 11-fold with ATP $\gamma \mathrm{S}$ as cofactor. Therefore UvsY appears to promote presynaptic filament assembly primarily by stabilizing a salt-sensitive pre-nucleation complex. Second, $k_{4}$ of the growth phase appears to be rate-limiting for UvsY-dependent filament assembly at high salt (avg. $k_{4} / k_{2}=0.08$ and 0.2 with ATP and ATP $\gamma \mathrm{S}$ as cofactor, 
respectively). The same is true for UvsY-independent reactions at low salt with ATP $\gamma \mathrm{S}$ as cofactor (avg. $k_{4} / k_{2}=0.2$ ). Third, in UvsY-independent reactions at low salt, ATP $\gamma \mathrm{S}$ enhances both $k_{2}$ of the nucleation phase and $K_{3}$ of the growth phase by $\sim 6$-fold compared to reactions with ATP as cofactor. The implications of these findings are discussed below.

\section{DISCUSSION}

Data from our ensemble measurements of T4 presynaptic filament assembly is described well by a relatively simple, nucleation-growth kinetic model (Figure 5). The model is similar to nucleation-growth models for RecA filament formation on dsDNA or SSB-ssDNA that were derived from single molecule measurements. ${ }^{19,25}$ The utility of the model is established by the fact that hyperbolic plots of observed rate constants vs. UvsX concentration (Fig. 3), and global fitting of model-derived differential equations to raw fluorescence traces (Fig. 4), both return similar values for kinetic parameters under almost all conditions (Table 3). Under one narrow set of conditions (intermediate UvsX concentrations in $200 \mathrm{mM} \mathrm{NaCl}$ plus UvsY and ATP $\gamma \mathrm{S}$ ), the global fitting algorithm failed to converge, suggesting additional complexity. One possible reason for the lack of convergence is a competition between the nucleation and growth phases for UvsX under these conditions. UvsY and ATP $\gamma$ S have been shown to stabilize UvsX-ssDNA interactions synergistically at high salt concentrations. ${ }^{8}$ It is conceivable that this leads to a burst of nucleation events that, at relatively low UvsX concentrations, depletes it from the growth phase. This would be consistent with the idea that UvsY promotes multiple nucleation events leading to many short filaments (see below).

\section{Mediator protein UvsY stabilizes a salt-sensitive pre-nucleation complex}

As illustrated in the model (Fig. 5), the critical first step $\left(K_{1}\right)$ of this mechanism is the ATPdependent formation of a pre-nucleation complex containing UvsX recombinase cooccupying the ssDNA along with the SSB component, Gp32. The pre-nucleation complex is distinguished from subsequent intermediates by its relative salt-sensitivity and by fluorescence enhancement effects on a Gp32F probe. It is worth noting that the T4 helicase loading protein, $\mathrm{Gp} 59$, enhances the fluorescence of Gp32F within a tripartite Gp59-Gp32FssDNA complex. ${ }^{26}$ The formation of this complex precedes loading of the replicative helicase, Gp41, which is accompanied by Gp32F displacement. The parallel fluorescence effects on Gp32F seen during UvsX loading and helicase loading suggest that transient intermediates in both processes may involve similar changes in Gp32-ssDNA structure or environment.

The data indicate that UvsY specifically stabilizes the pre-nucleation complex at high salt concentrations (Table 3; Figure 5). By overcoming the salt-sensitivity of presynaptic filament nucleation, UvsY activates UvsX to perform its recombination functions under physiological conditions. The observed salt-sensitivity of filament nucleation is consistent with the observation that the intrinsic affinity parameter of UvsX for ssDNA, $K_{\mathrm{ss}}$, is saltsensitive. ${ }^{21}$ The isolated binding of UvsX to ssDNA during filament nucleation presumably depends on $K_{\mathrm{ss}}$ alone, while contiguous binding of UvsX to ssDNA during filament growth also depends on $\omega$, the salt-insensitive cooperativity parameter. The salt-dependence of UvsX/Gp32 exchange also reflects differential salt effects on the ssDNA-binding activities of Gp32 and UvsX. Over the salt interval covered in this study (50-250 mM NaCl), Gp32ssDNA binding affinity is only weakly dependent on salt concentration; in fact the affinity decreases slightly at $50 \mathrm{mM} \mathrm{NaCl}$ (Table $1 ;{ }^{18,27}$ ). This is attributable to a masking effect caused by the acidic $\mathrm{C}$-terminal domain of $\mathrm{Gp} 32$, which has been well-documented elsewhere ${ }^{27}$ In contrast, the ssDNA-binding affinity of UvsX increases markedly with decreasing salt concentration over the same interval, and UvsX-ssDNA interactions are 
further stabilized by ATP binding. ${ }^{8}, 28$ Therefore at $50 \mathrm{mM} \mathrm{NaCl}$ UvsX-ATP possesses sufficient ssDNA-binding affinity to compete with Gp32 for binding sites on ssDNA, while at $200 \mathrm{mM} \mathrm{NaCl}$ it does not, and so requires the mediator activity of UvsY.

The observed salt-sensitivity of UvsX-ssDNA filament nucleation is consistent with results of single molecule studies of $E$. coli RecA-dsDNA filaments. The latter revealed a strong impact of ionic strength on nucleation cluster frequency, while the impact is much milder on the average cluster growth rate. ${ }^{25}$ Somewhat different results were obtained in single molecule studies of RecA filament assembly on SSB-ssDNA complexes, which found relatively modest effects of salt on filament nucleation and growth rates from 50-200 mM $\mathrm{NaCl} .{ }^{19}$ This difference could be due to the use of ATP $\gamma \mathrm{S}$ in the latter study, since ATP $\gamma \mathrm{S}$ greatly stabilizes RecA-ssDNA filaments in salt concentrations as high as $600 \mathrm{mM} \mathrm{NaCl},{ }^{29}$ which likely precludes the need for a mediator protein. In contrast, UvsX-ssDNA filaments in the presence of ATP (this study) are significantly more salt-sensitive, and therefore nucleation becomes mediator-dependent with increasing salt.

\section{Growth is rate-limiting in presynaptic filament assembly}

UvsX filament assembly on Gp32-ssDNA is characterized by relatively fast nucleation followed by relatively slow growth (Tables 2 and 3 ). The forward rate constant for the isomerization step of growth, $k_{4}$, appears to be rate-limiting under most conditions. This observation suggests that presynaptic filaments assemble via multiple nucleation events, forming many short filaments rather than a few long ones. The need for many nucleation events, along with the strict requirement for UvsY in filament nucleation at high salt, helps to explain the fact that UvsY is needed in approximately 1:1 stoichiometry with UvsX for optimal strand exchange activity. ${ }^{7,}{ }^{14}$ It is also consistent with single-molecule studies of human RAD51 and E. coli RecA assembly on duplex DNA, which show that these recombinases rapidly form multiple nucleation sites. ${ }^{25,30,31}$

Gp32 displacement from ssDNA by UvsX \pm UvsY proteins is a relatively slow process, yet presynaptic filament assembly occurs rapidly enough to account for observed rates of UvsXcatalyzed DNA strand exchange. At high UvsX concentrations in $50 \mathrm{mM} \mathrm{NaCl}+\mathrm{ATP}$, UvsX approaches half-saturation of the ssDNA within 1.5-2 min (Figure 4), which corresponds to the earliest times at which recombination intermediates appear in DNA strand exchange reactions performed under similar conditions. ${ }^{7,} 28$ The data suggest that partial saturation of ssDNA by UvsX protein is sufficient to initiate homologous pairing. At high UvsX concentrations in $200 \mathrm{mM} \mathrm{NaCl}+\mathrm{ATP}+\mathrm{UvsY}$, UvsX approaches halfsaturation of the ssDNA within 4-5 min (Figure 4). Early strand exchange and recombination-dependent replication products typically appear within 2-5 min under similar conditions, and require significantly lower concentrations of UvsX than are required under UvsY-independent conditions. ${ }^{7}, 13$ The data suggest that relatively short, UvsY-stabilized filaments are preferred to initiate homologous pairing reactions, which are not limited by slow filament growth rates.

\section{UvsX recombinase actively displaces Gp32 from ssDNA}

As illustrated in Figure 5, both nucleation and growth phases include isomerization steps $\left(k_{2}\right.$ and $k_{4}$ ) in which Gp32 is ejected from the ssDNA. In our experiments with the Gp32F probe, values of $k_{2}$ and $k_{4}$ (Table 3 ) greatly exceed values of the rate constant for spontaneous dissociation of Gp32F from cooperative clusters $\left(k_{-2}\right.$ in Table 1) under all conditions examined. Therefore UvsX does not passively occupy binding sites abandoned by Gp32; rather it actively displaces Gp32 from the lattice. Without the challenge from UvsX, pre-assembled Gp32F-ssDNA remains as a stable complex for over ten minutes without detectable dissociation (data not shown). Active displacement requires ATP (or 
ATP $\gamma$ S) binding by UvsX, and also requires UvsY under high-salt conditions. One possible mechanism for active displacement is shown schematically in Figure 5: We propose that isomerization of UvsX not only displaces Gp32 from ssDNA locally, but also remodels and destabilizes adjacent ends of Gp32 clusters, effectively increasing their off-rate from ssDNA.

\section{Implications for recombination in T4 and other systems}

Presynaptic filament assembly involves an intricate choreography of protein-ssDNA and protein-protein interactions among recombinase, SSB, and mediator components of the homologous recombination machinery. Each interaction is modulated by solution variables including ion concentrations and nucleotide ligands. These variables determine the efficiency of recombinase/SSB exchange on ssDNA and thereby dictate the requirement for mediator protein activities in filament assembly. In this study, we have quantified key aspects of the salt, nucleotide, and mediator protein effects that influence recombinase/SSB exchange during T4 presynaptic filament assembly. Results indicate that the efficiency of T4 presynaptic filament assembly in vivo is controlled by two major kinetic effects: (1) The requirement for UvsY mediator to stabilize the pre-nucleation complex formed by UvsX recombinase on Gp32-covered ssDNA; and (2) The rate-limiting nature of filament growth. The data suggest that $\mathrm{T} 4 u v s Y$ mutant strains are recombination-deficient specifically because they cannot form UvsX pre-nucleation complexes under in vivo salt conditions. Our model predicts that it should be possible to isolate UvsX or Gp32 mutants, or to identify chemical agents, that suppress $u v s Y$ mutations by stabilizing the pre-nucleation complex. In this regard, a yeast Rad51-I345T mutant protein shows enhanced binding and stability to ssand ds-DNA, which can partially bypass the requirement for Rad55-Rad57 mediator proteins in DNA repair. ${ }^{32}$ Conversely, agents that destabilize the pre-nucleation complex would be predicted to inhibit recombination in vivo.

Presynaptic filament structure and function are highly conserved among diverse species; likewise SSB and mediator proteins are functionally conserved. Therefore it seems probable that cellular recombination systems follow some of the same kinetic principles as those employed by the T4 system. Our kinetic studies suggest that UvsX/UvsY form many nucleation centers and short segments on Gp32-ssDNA, and the dominance of filament nucleation argues for a kinetically-competent discontinuous filament. A growing body of evidence suggests that discontinuous presynaptic filaments may be a general feature of all homologous recombination systems. ${ }^{36}$ Single-molecule studies have demonstrated that RecA/Rad51 form multiple nucleation sites on either naked or SSB-covered DNA. ${ }^{19,25,30,33}$ Rad51 forms protein patches with gaps on dsDNA molecules and typically forms irregular structures on ssDNA. ${ }^{30,34}$ EM structures of human Rad51-Rad52 cofilaments reveal multiple individual Rad52 binding sites on Rad51-covered ssDNA. ${ }^{35}$ $\operatorname{Rad} 51$ paralogs Rad55/Rad57 in yeast, and $\operatorname{Rad} 51 \mathrm{~B} / \operatorname{Rad} 51 \mathrm{C}$ in humans, both appear to integrate into Rad51-ssDNA presynaptic filaments and protect them from disruption by antirecombination helicases Srs 2 and Blm, respectively. ${ }^{37,38}$ We propose that the formation of discontinuous filaments may be advantageous for their regulation by recombination mediator proteins, both at the level of nucleation (e.g. by classic mediators such as T4 UvsY, E. coli $\mathrm{RecFOR}, \operatorname{Rad} 52$, or Brca2 ${ }^{4,36}$ ), and at the level of segment stabilization (e.g. by Rad51 paralogs such as $\operatorname{Rad} 55 / \operatorname{Rad} 57$ or $\operatorname{Rad} 51 \mathrm{~B} / \operatorname{Rad} 51 \mathrm{C})$. It seems possible that in humans the Rad51-ssDNA filament could be deliberately managed by Brca2 and Rad51B/Rad51C to form relatively short, stable segments that are optimized to promote recombination by a synthesis-dependent strand annealing (SDSA) mechanism that avoids genotoxic crossovers and genomic instability. ${ }^{39}$ Bacteriophage T4 is proficient in SDSA, which is promoted in vitro by purified UvsX, UvsY, Gp32, and replication enzymes. ${ }^{40}$ Mechanistic studies of this 
process are likely to inform studies of SDSA and related genome stability processes in humans and other organisms.

\section{References}

1. Bianco PR, Tracy RB, Kowalczykowski SC. DNA strand exchange proteins: a biochemical and physical comparison. Front Biosci. 1998; 3:570-603.

2. Khanna KK, Jackson SP. DNA double-strand breaks: signaling, repair and the cancer connection. Nat Genet. 2001; 27:247-254. [PubMed: 11242102]

3. Jasin M. Homologous repair of DNA damage and tumorigenesis: the BRCA connection. Oncogene. 2002; 21:8981-8993. [PubMed: 12483514]

4. Beernink HT, Morrical SW. RMPs: recombination/replication mediator proteins. Trends Biochem Sci. 1999; 24:385-389. [PubMed: 10500302]

5. Bleuit JS, Xu H, Ma Y, Wang T, Liu J, Morrical SW. Mediator proteins orchestrate enzyme-ssDNA assembly during T4 recombination-dependent DNA replication and repair. Proc Natl Acad Sci U S A. 2001; 98:8298-8305. [PubMed: 11459967]

6. Liu J, Qian N, Morrical SW. Dynamics of bacteriophage T4 presynaptic filament assembly from extrinsic fluorescence measurements of Gp32-single-stranded DNA interactions. J Biol Chem. 2006b; 281:26308-26319. [PubMed: 16829679]

7. Bleuit JS, Ma Y, Munro J, Morrical SW. Mutations in a conserved motif inhibit single-stranded DNA binding and recombination mediator activities of bacteriophage T4 UvsY protein. J Biol Chem. 2004; 279:6077-6086. [PubMed: 14634008]

8. Liu J, Bond JP, Morrical SW. Mechanism of presynaptic filament stabilization by the bacteriophage T4 UvsY recombination mediator protein. Biochemistry. 2006a; 45:5493-5502. [PubMed: 16634631]

9. Sweezy MA, Morrical SW. Biochemical interactions within a ternary complex of the bacteriophage T4 recombination proteins uvsY and gp32 bound to single-stranded DNA. Biochemistry. 1999; 38:936-944. [PubMed: 9893989]

10. Kodadek T, Gan DC, Stemke-Hale K. The phage T4 uvsY recombination protein stabilizes presynaptic filaments. J Biol Chem. 1989; 264:16451-16457. [PubMed: 2550444]

11. Harris LD, Griffith JD. UvsY protein of bacteriophage T4 is an accessory protein for in vitro catalysis of strand exchange. J Mol Biol. 1989; 206:19-27. [PubMed: 2522995]

12. Yonesaki T, Minagawa T. Synergistic action of three recombination gene products of bacteriophage T4, uvsX, uvsY, and gene 32 proteins. J Biol Chem. 1989; 264:7814-7820. [PubMed: 2785988]

13. Morrical SW, Alberts BM. The UvsY protein of bacteriophage T4 modulates recombinationdependent DNA synthesis in vitro. J Biol Chem. 1990; 265:15096-15103. [PubMed: 2144282]

14. Yassa DS, Chou KM, Morrical SW. Characterization of an amino-terminal fragment of the bacteriophage T4 uvsY recombination protein. Biochimie. 1997; 79:275-285. [PubMed: 9258436]

15. Formosa T, Alberts BM. Purification and characterization of the T4 bacteriophage uvsX protein. $\mathrm{J}$ Biol Chem. 1986; 261:6107-6118. [PubMed: 2939071]

16. Cunningham RP, Berger H. Mutations affecting genetic recombination in bacteriophage T4D. I. Pathway analysis. Virology. 1977; 80:67-82. [PubMed: 878316]

17. Melamede RJ, Wallace SS. Properties of the nonlethal recombinational repair $\mathrm{x}$ and $\mathrm{y}$ mutants of bacteriophage T4. II. DNA synthesis. Journal of virology. 1977; 24:28-40. [PubMed: 904025]

18. Lohman TM, Kowalczykowski SC. Kinetics and mechanism of the association of the bacteriophage T4 gene 32 (helix destabilizing) protein with single-stranded nucleic acids. Evidence for protein translocation. J Mol Biol. 1981; 152:67-109. [PubMed: 6279865]

19. Bell JC, Plank JL, Dombrowski CC, Kowalczykowski SC. Direct imaging of RecA nucleation and growth on single molecules of SSB-coated ssDNA. Nature. 2012; 491:274-278. [PubMed: 23103864]

20. Kuzmic P. Program DYNAFIT for the analysis of enzyme kinetic data: application to HIV proteinase. Anal Biochem. 1996; 237:260-273. [PubMed: 8660575] 
21. Ando RA, Morrical SW. Single-stranded DNA binding properties of the UvsX recombinase of bacteriophage T4: binding parameters and effects of nucleotides. J Mol Biol. 1998; 283:785-796. [PubMed: 9790840]

22. Farb JN, Morrical SW. Role of allosteric switch residue histidine 195 in maintaining active-site asymmetry in presynaptic filaments of bacteriophage T4 UvsX recombinase. J Mol Biol. 2009a; 385:393-404. [PubMed: 19027026]

23. Maher RL, Morrical SW. Coordinated binding of single-stranded and double-stranded DNA by UvsX recombinase. PLoS ONE. 2013; 8:e66654. [PubMed: 23824136]

24. Sweezy MA, Morrical SW. Single-stranded DNA binding properties of the uvsY recombination protein of bacteriophage T4. J Mol Biol. 1997; 266:927-938. [PubMed: 9086271]

25. Galletto R, Amitani I, Baskin RJ, Kowalczykowski SC. Direct observation of individual RecA filaments assembling on single DNA molecules. Nature. 2006; 443:875-878. [PubMed: 16988658]

26. Branagan AM, Maher RL, Morrical SW. Assembly and dynamics of Gp59-Gp32-single-stranded DNA (ssDNA), a DNA helicase loading complex required for recombination-dependent replication in bacteriophage T4. The Journal of biological chemistry. 2012; 287:19070-19081. [PubMed: 22500043]

27. Pant K, Karpel RL, Rouzina I, Williams MC. Salt dependent binding of T4 gene 32 protein to single and double-stranded DNA: single molecule force spectroscopy measurements. J Mol Biol. 2005; 349:317-330. [PubMed: 15890198]

28. Kodadek T, Wong ML, Alberts BM. The mechanism of homologous DNA strand exchange catalyzed by the bacteriophage T4 uvsX and gene 32 proteins. J Biol Chem. 1988; 263:94279436. [PubMed: 2967823]

29. Menetski JP, Kowalczykowski SC. Interaction of recA protein with single-stranded DNA. Quantitative aspects of binding affinity modulation by nucleotide cofactors. J Mol Biol. 1985; 181:281-295. [PubMed: 3981638]

30. Modesti M, Ristic D, van der Heijden T, Dekker C, van Mameren J, Peterman EJ, Wuite GJ, Kanaar R, Wyman C. Fluorescent human RAD51 reveals multiple nucleation sites and filament segments tightly associated along a single DNA molecule. Structure. 2007; 15:599-609. [PubMed: 17502105]

31. van der Heijden T, Seidel R, Modesti M, Kanaar R, Wyman C, Dekker C. Real-time assembly and disassembly of human RAD51 filaments on individual DNA molecules. Nucleic acids research. 2007; 35:5646-5657. [PubMed: 17709342]

32. Fortin GS, Symington LS. Mutations in yeast Rad51 that partially bypass the requirement for Rad55 and Rad57 in DNA repair by increasing the stability of Rad51-DNA complexes. The EMBO journal. 2002; 21:3160-3170. [PubMed: 12065428]

33. Hilario J, Amitani I, Baskin RJ, Kowalczykowski SC. Direct imaging of human Rad51 nucleoprotein dynamics on individual DNA molecules. Proc Natl Acad Sci U S A. 2009; 106:361368. [PubMed: 19122145]

34. Ristic D, Modesti M, van der Heijden T, van Noort J, Dekker C, Kanaar R, Wyman C. Human Rad51 filaments on double- and single-stranded DNA: correlating regular and irregular forms with recombination function. Nucleic acids research. 2005; 33:3292-3302. [PubMed: 15944450]

35. Van Dyck E, Hajibagheri NM, Stasiak A, West SC. Visualisation of human rad52 protein and its complexes with hRad51 and DNA. J Mol Biol. 1998; 284:1027-1038. [PubMed: 9837724]

36. Liu J, Ehmsen KT, Heyer WD, Morrical SW. Presynaptic filament dynamics in homologous recombination and DNA repair. Crit Rev Biochem Mol Biol. 2011; 46:240-270. [PubMed: 21599536]

37. Liu J, Renault L, Veaute X, Fabre F, Stahlberg H, Heyer WD. Rad51 paralogues Rad55-Rad57 balance the antirecombinase Srs2 in Rad51 filament formation. Nature. 2011; 479:245-248. [PubMed: 22020281]

38. Amunugama R, Groden J, Fishel R. The HsRAD51B-HsRAD51C stabilizes the HsRAD51 nucleoprotein filament. DNA Repair (Amst). 2013; 12:723-732. [PubMed: 23810717] 
39. Maher RL, Branagan AM, Morrical SW. Coordination of DNA replication and recombination activities in the maintenance of genome stability. J Cell Biochem. 2011; 112:2672-2682. [PubMed: 21647941]

40. Liu J, Morrical SW. Assembly and dynamics of the bacteriophage T4 homologous recombination machinery. Virol J. 2010; 7:357. [PubMed: 21129202] 

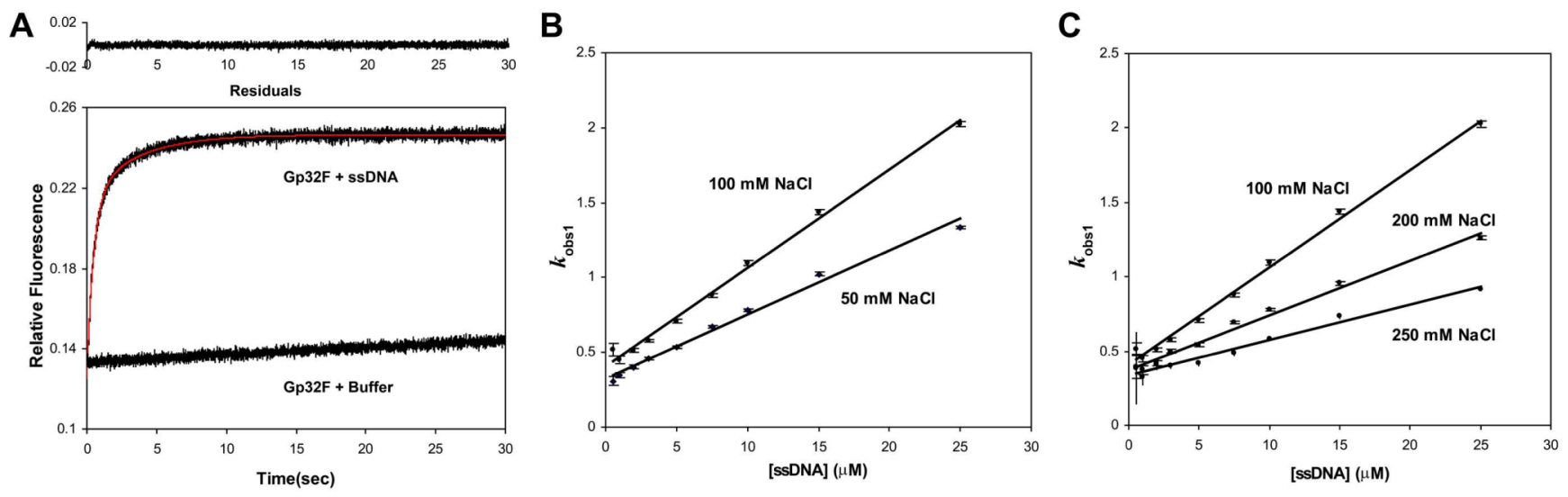

Figure 1.

(A) Time course of rapid association between $25 \mu \mathrm{M}$ M13mp18 ssDNA and $0.5 \mu \mathrm{M}$ Gp32F, shown in black. The red curve represents the double exponential fit of the data according to Eq. $1\left(k_{\mathrm{obs} 1}=2.02 \pm 0.02 \mathrm{~s}^{-1}\right.$ and $\left.k_{\mathrm{obs} 2}=0.292 \pm 0.003 \mathrm{~s}^{-1}\right)$, and the residuals are shown in black at the top. The buffer control with only $0.5 \mu \mathrm{M} \mathrm{Gp32F}$ is shown in the black trace on the bottom, containing Buffer A with $100 \mathrm{mM} \mathrm{NaCl}, \mathrm{T}=25^{\circ} \mathrm{C}$. (B-C) Gp32F-M13mp18 ssDNA association as a function of $[\mathrm{NaCl}]$ under both strong $(\mathrm{B})$ and weak $(\mathrm{C})$ binding conditions. All the reactions in (B) and (C) contained $0.5 \mu \mathrm{M} \mathrm{Gp} 32 \mathrm{~F}$ and various amounts of M13mp18 ssDNA in Buffer A with indicated [ $\mathrm{NaCl}]$. The kinetic parameters from fitting (see text) are summarized in Table 1, which reflect the apparent on and off rates in the second step in the Lohman model, as shown in Scheme 1. ${ }^{18}$ 

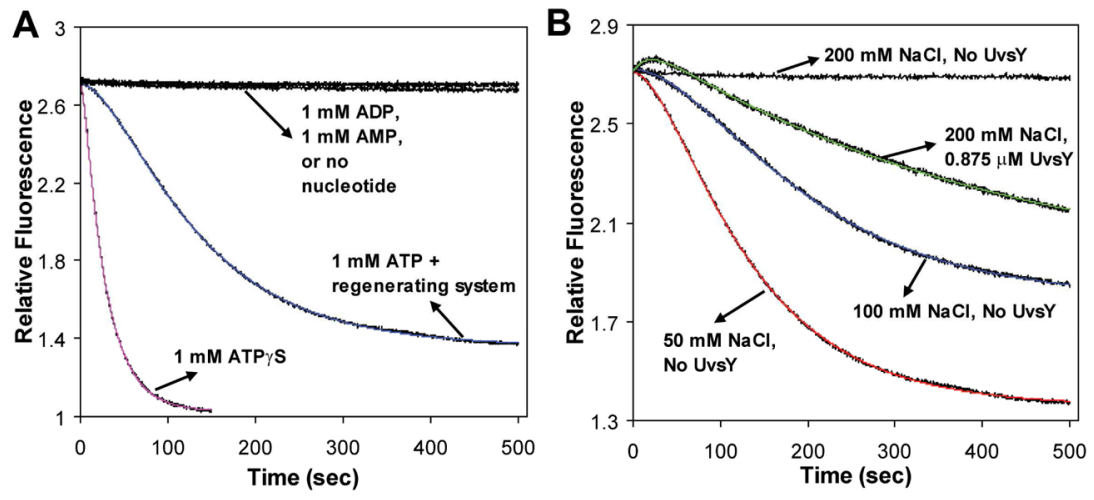

Figure 2.

The displacement of Gp32F from ssDNA by UvsX is affected by nucleotides, salt, and UvsY. (A) $0.5 \mu \mathrm{M}$ Gp32F - $3.5 \mu \mathrm{M}$ M13mp18 ssDNA complex was rapidly mixed with $0.875 \mu \mathrm{M}$ UvsX in Buffer $\mathrm{A}+50 \mathrm{mM} \mathrm{NaCl}$ and either no nucleotide, $1 \mathrm{mM}$ AMP, $1 \mathrm{mM}$ $\mathrm{ADP}, 1 \mathrm{mM}$ ATP + regenerating system (R.S.), or $1 \mathrm{mM} \mathrm{ATP} \gamma \mathrm{S}, \mathrm{T}=25^{\circ} \mathrm{C}$. Red and blue curves represent the best fits of Eq. 4 to reactions with $1 \mathrm{mM} \mathrm{ATP}+\mathrm{R} . \mathrm{S}$. and $1 \mathrm{mM} \mathrm{ATP} \gamma \mathrm{S}$, respectively. (B) $0.5 \mu \mathrm{M}$ Gp32F - $3.5 \mu \mathrm{M}$ M13mp18 ssDNA complex was rapidly mixed with $0.875 \mu \mathrm{M}$ UvsX $\pm 0.875 \mu \mathrm{M}$ UvsY in Buffer A + 1mM ATP + R.S. + either $50 \mathrm{mM}$, $100 \mathrm{mM}$, or $200 \mathrm{mM} \mathrm{NaCl}, \mathrm{T}=25^{\circ} \mathrm{C}$. Red, blue, and green curves represent the best fits of Eq. 4 to reactions with $50 \mathrm{mM} \mathrm{NaCl}, 100 \mathrm{mM} \mathrm{NaCl}$, or $200 \mathrm{mM} \mathrm{NaCl}+$ UvsY, respectively. Fitting parameters are shown in Table 2. 

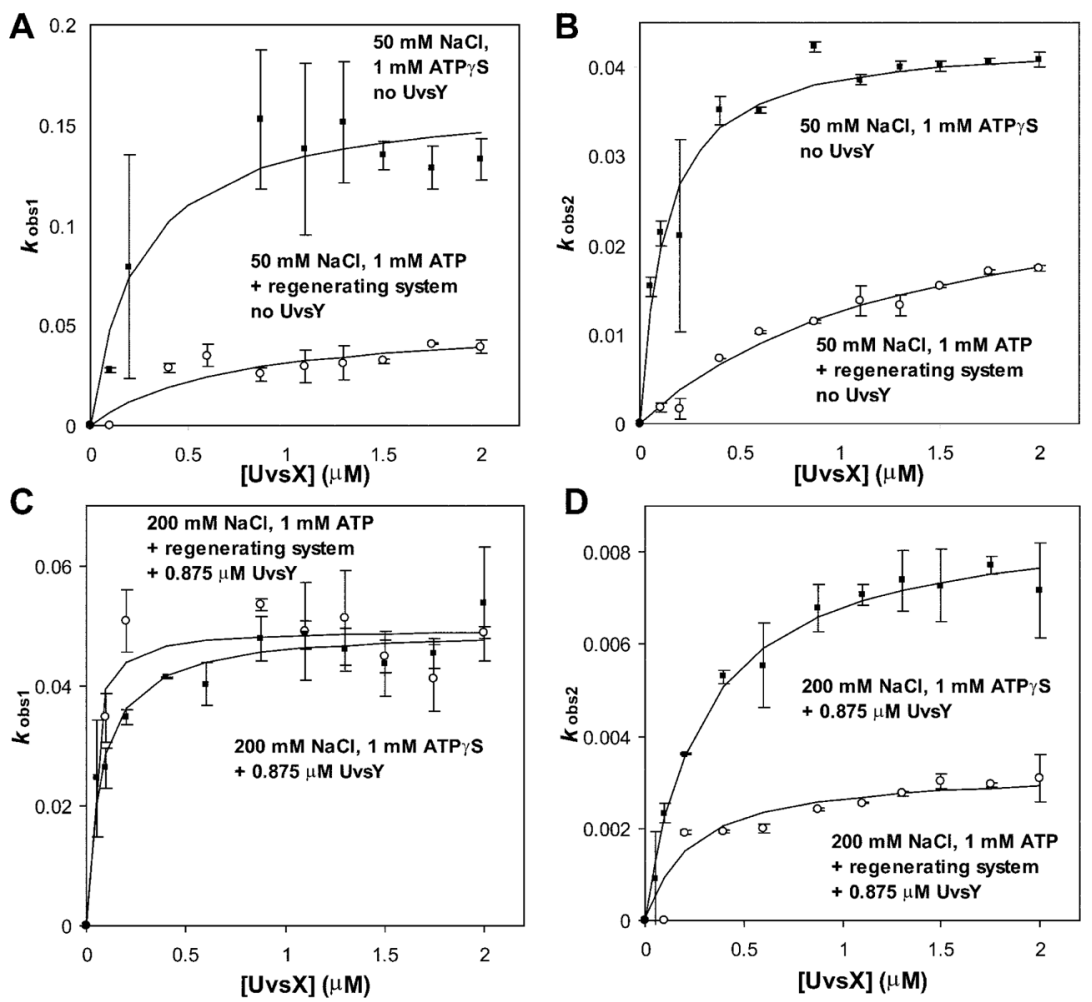

Figure 3.

Pre-steady state studies of UvsX-ssDNA binding via Gp32F displacement. (A-B) Observed rate constants of (A) UvsX nucleation $\left(k_{\mathrm{obs} 1}\right)$ and (B) UvsX growth $\left(k_{\mathrm{obs} 2}\right)$ on Gp32FssDNA as functions of [UvsX] in Buffer A + $50 \mathrm{mM} \mathrm{NaCl}+$ either $1 \mathrm{mM}$ ATP + R.S., or 1 $\mathrm{mM} A T P \gamma S$ as indicated. For all reactions in (A) and (B), $0.5 \mu \mathrm{M} \mathrm{Gp} 32 \mathrm{~F}-3.5 \mu \mathrm{M}$

M13mp18 ssDNA complex was rapidly mixed with indicated amount of UvsX at $\mathrm{T}=25^{\circ} \mathrm{C}$. (C-D) Observed rate constants of (C) UvsX nucleation $\left(k_{\mathrm{obs} 1}\right)$ and (D) UvsX propogation $\left(k_{\mathrm{obs} 2}\right)$ on Gp32F-ssDNA in the presence of UvsY, as a function of [UvsX] in Buffer A + $200 \mathrm{mM} \mathrm{NaCl}+$ either $1 \mathrm{mM}$ ATP + R.S., or $1 \mathrm{mM} \mathrm{ATP} \gamma \mathrm{S}$ as indicated. For all reactions in (C) and (D), $0.5 \mu \mathrm{M}$ Gp32F - $3.5 \mu \mathrm{M}$ M13mp18 ssDNA complex was rapidly mixed with indicated amount of UvsX $+0.875 \mu \mathrm{M}$ Uvs $\mathrm{Y}$ at $\mathrm{T}=25^{\circ} \mathrm{C}$. Curves in (A) - (D) represent fits of the data to Eqs. 2-3. Fitting parameters are shown in Table 3. 
A
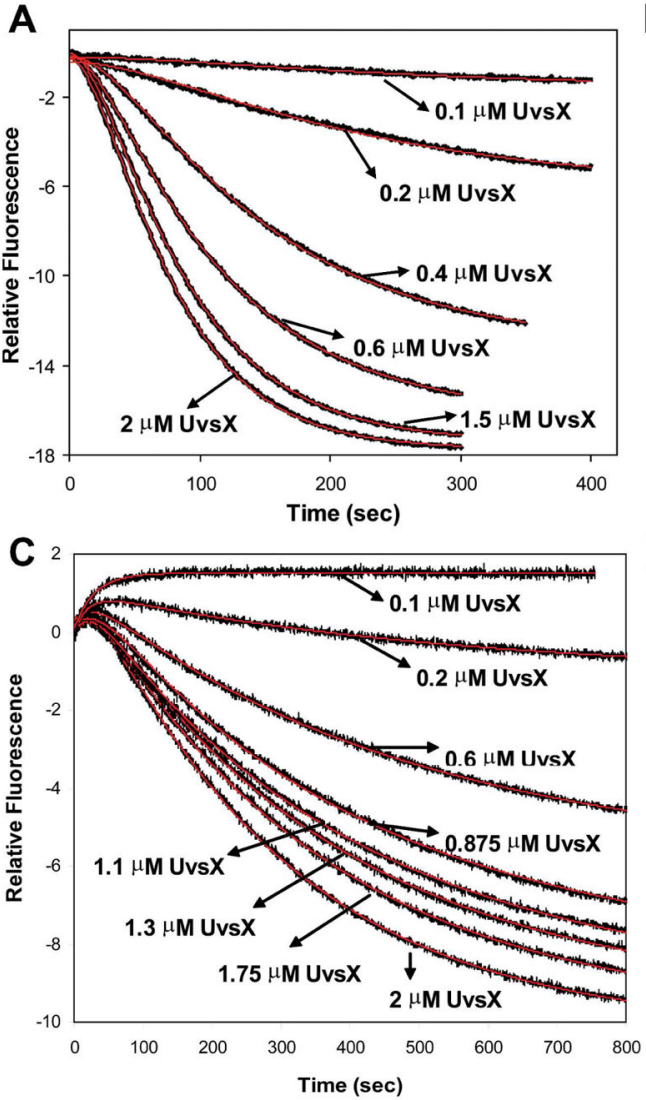

B

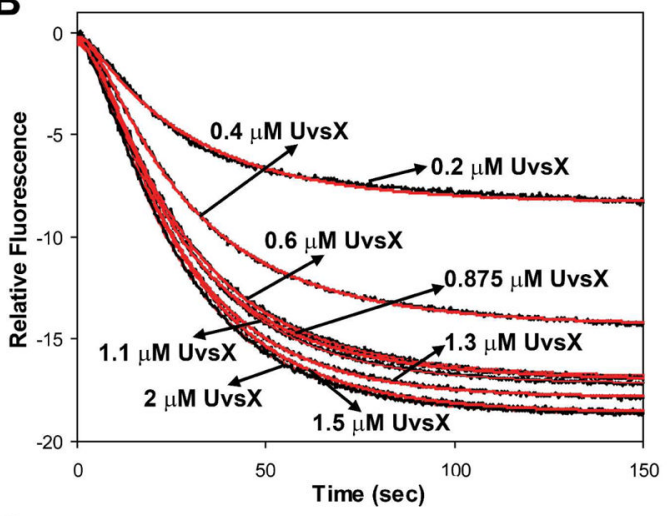

D

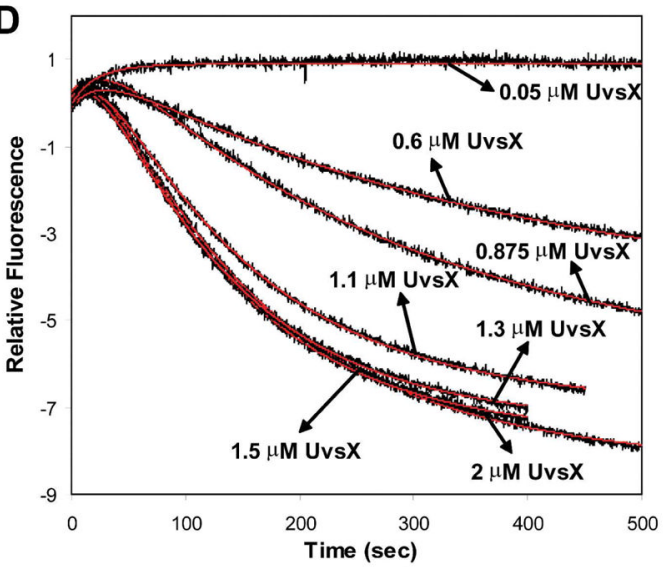

Figure 4.

Time courses of Gp32F displacement by different concentrations of UvsX. Red curves represents the best stepwise global fit models (Eq. 7). Fitting parameters are shown in Table 3. (A-B) Reactions in Buffer A + $50 \mathrm{mM} \mathrm{NaCl}+$ either (A) $1 \mathrm{mM}$ ATP + R.S. or (B) $1 \mathrm{mM}$ ATP $\gamma$ S. For all reactions in (A) and (B), $0.5 \mu \mathrm{M}$ Gp32F - 3.5 $\mu \mathrm{M}$ M13mp18 ssDNA complex was rapidly mixed with indicated amount of UvsX at $\mathrm{T}=25^{\circ} \mathrm{C}$. (C-D) Reactions in Buffer A + $200 \mathrm{mM} \mathrm{NaCl}+0.875 \mu \mathrm{M}$ UvsY + either $1 \mathrm{mM} \mathrm{ATP}+$ R.S. (C) or $1 \mathrm{mM}$ ATP $\gamma \mathrm{S}(\mathrm{D})$. For all reactions in (C) and (D), $0.5 \mu \mathrm{M}$ Gp32F - $3.5 \mu \mathrm{M}$ M13mp18 ssDNA complex was rapidly mixed with indicated amount of UvsX $+0.875 \mu \mathrm{M} \mathrm{UvsY}$ at $\mathrm{T}=25^{\circ} \mathrm{C}$. During curve fitting, iterative runs for individual isotherms were taken to determine values and standard errors of kinetic constants. 


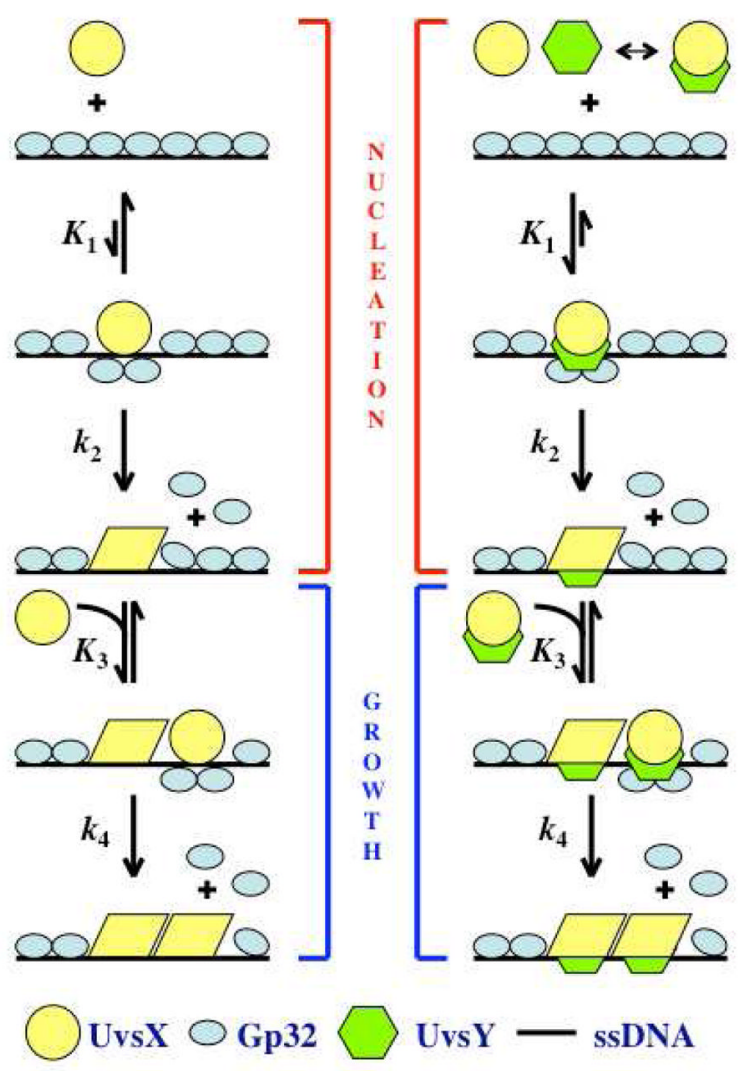

Figure 5.

A model for UvsX presynaptic filament assembly on Gp32-covered ssDNA. Left - Under low-salt conditions in the absence of mediator protein UvsY, ATP-bound UvsX, a high affinity form, binds Gp32-ssDNA rapidly to form an unstable nucleation site or "prenucleation complex" (association constant $K_{1}$ ). A slow but almost irreversible conformational change (forward rate constant $k_{2}$ ) is required by UvsX to displace Gp32 and to secure this isolated nucleation site on the lattice. With successful nucleation, more ATPbound UvsX is recruited to form an unstable cluster (association constant $K_{3}$ ). This rapidly formed UvsX cluster undergoes another slow but almost irreversible conformational change to displace $\mathrm{Gp} 32$ and to redistribute into a stable and productive presynaptic filament (forward rate constant $k_{4}$ ). Right - Under high-salt conditions the mediator protein, UvsY, facilitates filament nucleation by stabilizing the salt-sensitive pre-nucleation complex (enhanced $K_{1}$ ), by forming a special quaternary complex with UvsX, Gp32, and ssDNA. Filament growth (particularly $k_{4}$ ) is rate-limiting under both UvsY-dependent and independent conditions. Therefore filament assembly necessary for DNA strand exchange and recombination functions of UvsX likely involves frequent nucleation events leading to many short filaments. See text for details. 


$$
\begin{aligned}
& \mathrm{Gp} 32 \mathrm{~F}+\mathrm{ssDNA} \underset{k_{1}}{\stackrel{k_{1}}{\rightleftharpoons} \mathrm{Gp} 32 \mathrm{~F} \bullet \operatorname{ssDNA}_{\text {isolated }} \quad \text { Step 1: Nucleation }} \\
& \mathrm{Gp} 32 \mathrm{~F} \bullet \mathrm{ssDNA}_{\text {isolated }}+\mathrm{Gp} 32 \mathrm{~F} \underset{k_{-2}}{\stackrel{k_{2}}{k_{-1}}} \mathrm{Gp} 32 \mathrm{~F} \bullet \mathrm{ssDNA}_{\text {contiguous }} \quad \text { Step 2: Growth } \\
& \mathrm{Gp} 32 \mathrm{~F} \bullet \mathrm{ssDNA}_{\text {contiguous }} \stackrel{k_{\tau}}{\longrightarrow} \mathrm{Gp} 32 \mathrm{~F} \bullet \mathrm{ssDNA}_{\text {equilibrium cluster redistribution }} \text { Step 3: Isomerization }
\end{aligned}
$$

Scheme 1.

Model for Gp32F-ssDNA association kinetics. 


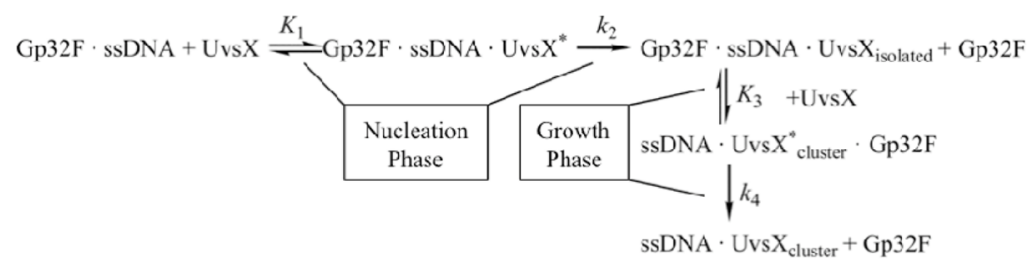

Scheme 2.

Nucleation-growth kinetic model for UvsX presynaptic filament assembly on Gp32Fcovered ssDNA. 


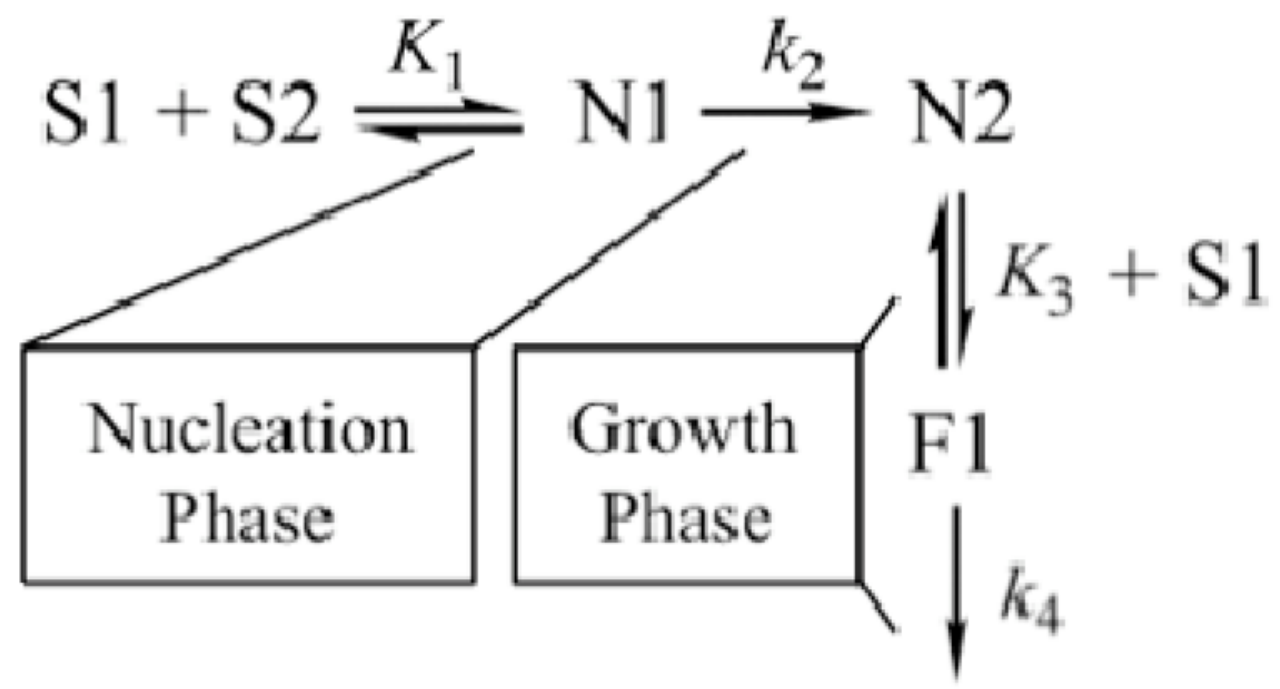

F2

Scheme 3.

Symbolic representation of Scheme 2, used for global fitting of model-derived differential equations. Scheme 3 is identical to Scheme 2 but contains the following term substitutions to facilitate mathematical modeling: [S1] represents the concentration of free UvsX protein; [S2] represents the concentration of Gp32F-covered ssDNA which is converted to all available binding sites for UvsX, according to a binding site size $n=4$ nucleotide residues; ${ }^{21}$ [N1] represents the concentration of an initial pre-nucleation complex Gp32F•ssDNA $\bullet \mathrm{Uvs}^{*}$ in which UvsX binds Gp32F-covered ssDNA to form isolated sites; [N2] represents the concentration of $\mathrm{Gp} 32 \mathrm{~F} \bullet \mathrm{ssDNA} \bullet \mathrm{Uvs}_{\mathrm{isolated}}$, an isomerized productive form of nucleation complex in which UvsX forms isolated stable sites on Gp32F-covered ssDNA; [F1] represents the concentration of a filament intermediate ssDNA $\bullet \mathrm{UvsX}^{*}{ }_{\text {Cluster }} \cdot \mathrm{Gp} 32 \mathrm{~F}$ in which UvsX forms initial clusters on Gp32F-covered ssDNA; [F2] represents the concentration of the isomerized productive cluster ssDNA $\cdot \mathrm{UvS}_{\mathrm{Cluster}}$ in which UvsX forms a productive presynaptic filament on ssDNA. 
Table 1

Kinetic parameters of $\mathrm{Gp} 32 \mathrm{~F}$ binding to ssDNA: Effects of $\mathrm{NaCl}$ concentration.

\begin{tabular}{lcccc}
\hline & \multicolumn{1}{c}{ Gp32F } & \multicolumn{2}{c}{ Gp32 (from ref. $\left.{ }^{\mathbf{1 8}}\right)$} \\
\hline$[\mathbf{N a C l}]$ & $\boldsymbol{k}_{\mathbf{2}}(\mathbf{a p p})\left(\mathbf{M}^{\mathbf{- 1}} \mathbf{s}^{\mathbf{- 1}}\right)$ & $\boldsymbol{k}_{\mathbf{- 2}}(\mathbf{a p p})\left(\mathbf{s}^{\mathbf{- 1}}\right)$ & $\boldsymbol{K}_{\mathbf{2}}\left(\mathbf{M}^{\mathbf{- 1}}\right)$ & $\boldsymbol{k}_{\mathbf{2}}\left(\mathbf{M}^{\mathbf{- 1}} \mathbf{s}^{\mathbf{- 1}}\right)$ \\
\hline $50 \mathrm{mM}$ & $4.3 \pm 0.2 * 10^{4}$ & $0.33 \pm 0.02$ & $1.3 * 10^{5}$ & $1.3 * 10^{6}$ \\
$100 \mathrm{mM}$ & $6.6 \pm 0.2 * 10^{4}$ & $0.41 \pm 0.02$ & $1.6^{*} 10^{5}$ & $2.8 * 10^{6}$ \\
$200 \mathrm{mM}$ & $3.7 \pm 0.1 * 10^{4}$ & $0.37 \pm 0.02$ & $1.0^{*} 10^{5}$ & --- \\
$250 \mathrm{mM}$ & $2.4 \pm 0.1 * 10^{4}$ & $0.34 \pm 0.02$ & $7.1 * 10^{4}$ & --- \\
\hline
\end{tabular}

The apparent rate constants $k_{2}$ and $k_{-2}$ and the calculated equilibrium constant $K_{2}$ for Gp32F-ssDNA interactions were derived from linear fits of the data in Figure 1B-C as described in Supporting Materials and Methods. Previously published values of $k 2$ for interactions of unmodified Gp32 with a poly $(\mathrm{r} \& \mathrm{~A})$ lattice are shown for comparison. 


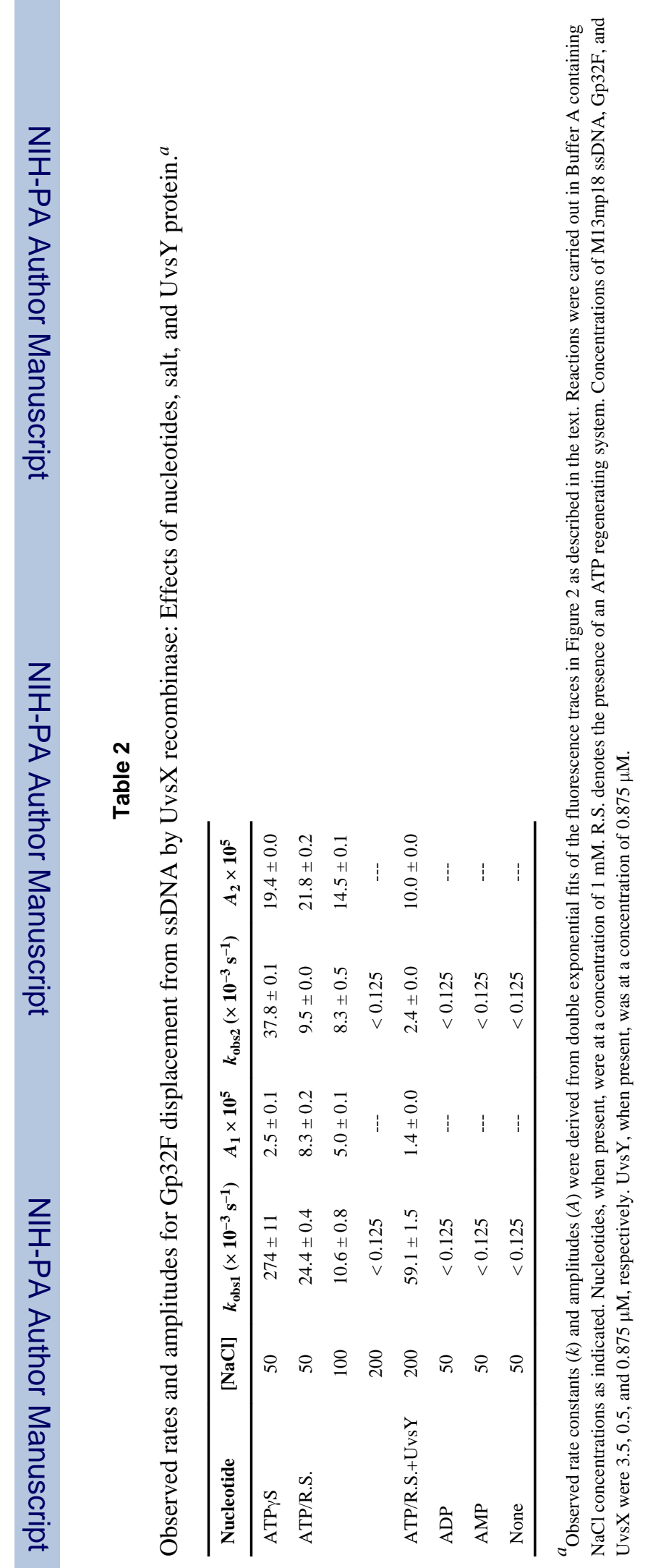

Biochemistry. Author manuscript; available in PMC 2014 November 12. 


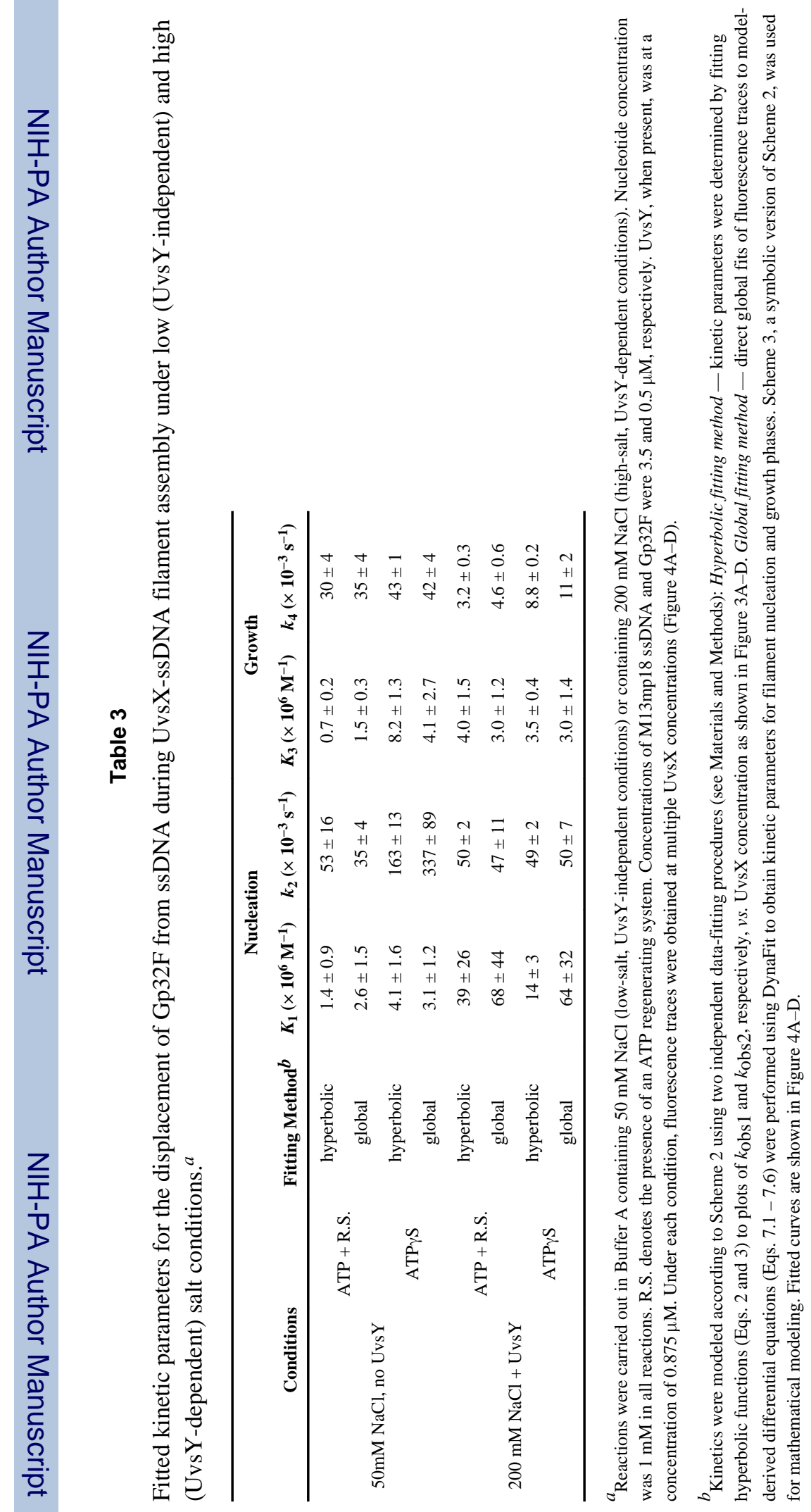

Biochemistry. Author manuscript; available in PMC 2014 November 12. 